\title{
Trazodone Not Only A Stabilizer in Allergic Rhinitis
}

\author{
Laura Buratti*, Chiara Rocchi, Viviana Totaro and Mauro Silvestrini \\ Neurological Clinic, Marche Polytechnic University, Italy \\ *Corresponding author: Laura Buratti, Neurological Clinic, Marche Polytechnic University, Via Conca 71, 60020 Ancona, Italy
}

Keywords: Obstructive sleep apnea; flow limitation; allergic rhinitis; trazodone; polygraphy

\section{Letter to Editor}

We report the case of a 44 years old woman referred to the outpatient Sleep Disorder Service of the Neurological Clinic, Marche Polytechnic University for a symptomatology characterized by difficulty in maintaining sleep and a feeling of unrefreshing sleep on waking. On clinical history, she referred an allergic rhinitis, treated with short cycles of inalatory steroids and vasoconstrictors. At the moment of our observation, she did not assume any treatment [1]. Due to the reported symptoms, particular attention was paid to the possible presence of sleep disturbances, in particular sleep-related breathing disorders. In this respect, patient reported a morning dry mouth. Further, the partner referred an important snoring and continuous lightening of sleep. Nocturnal apnea events were not reported as well as discomfort or abnormal movements in the legs. No other disturbance suggestive for NREM or REM sleep disturbances, nor fewer specific phases were evidenced. Patient was then submitted to a polygraphy which showed a rhonchopathy associated with air flow limitations, a high arousal index and elevated heart rate variability. The exam excluded periodic limb movements (Figure 1) [2,3]. Considering the history of allergic rhinitis and also to evaluate the possible presence of anomalies of the upper airways, an ENT assessment was indicated. Further, in relation to the difficulty in maintaining sleep and for the high indices of autonomic dysfunction, a pharmacological treatment with trazodone at the dose of sleep stabilizer, $30 \mathrm{mg}$ in the evening was prescribed.

ENT evaluation showed a pattern of nasal congestion without other alteration of the upper airways. Specific topical therapy was prescribed, not taken by the patient for the improvement with trazodone. Infact after few days of treatment with trazodone the patient reported a significant improvement of symptomatology and after 30 days a poligraphic control showed a complete recovery of respiratory alterations and a normalization of autonomic dysfunction (Figure 2) [4]. The particularly favorable and rapid evolution of patient's symptomatology could be interpreted on the basis of the pharmacological properties of trazodone. It is possible to hypothesize that trazodone at low dosages, in addition to the anxiolytic and hypno-inducing effect, for its an antihistamine action was able to contemporary solve clinical pictures related to both insomnia and the respiratory dysfunction [5]. Therefore, trazodone, in addition to stabilizing the sleep structure by increasing the arousal threshold, should deserve consideration in patients affected by allergic rhinitis with sleep disturbances. The advantage of having a valid therapeutic alternative would be very important in relation to the fact that steroids and vasoconstrictors, normally used in the treatment of allergic rhinitis, find an indication only in the short-term approach and have no effect on sleep disturbances. 


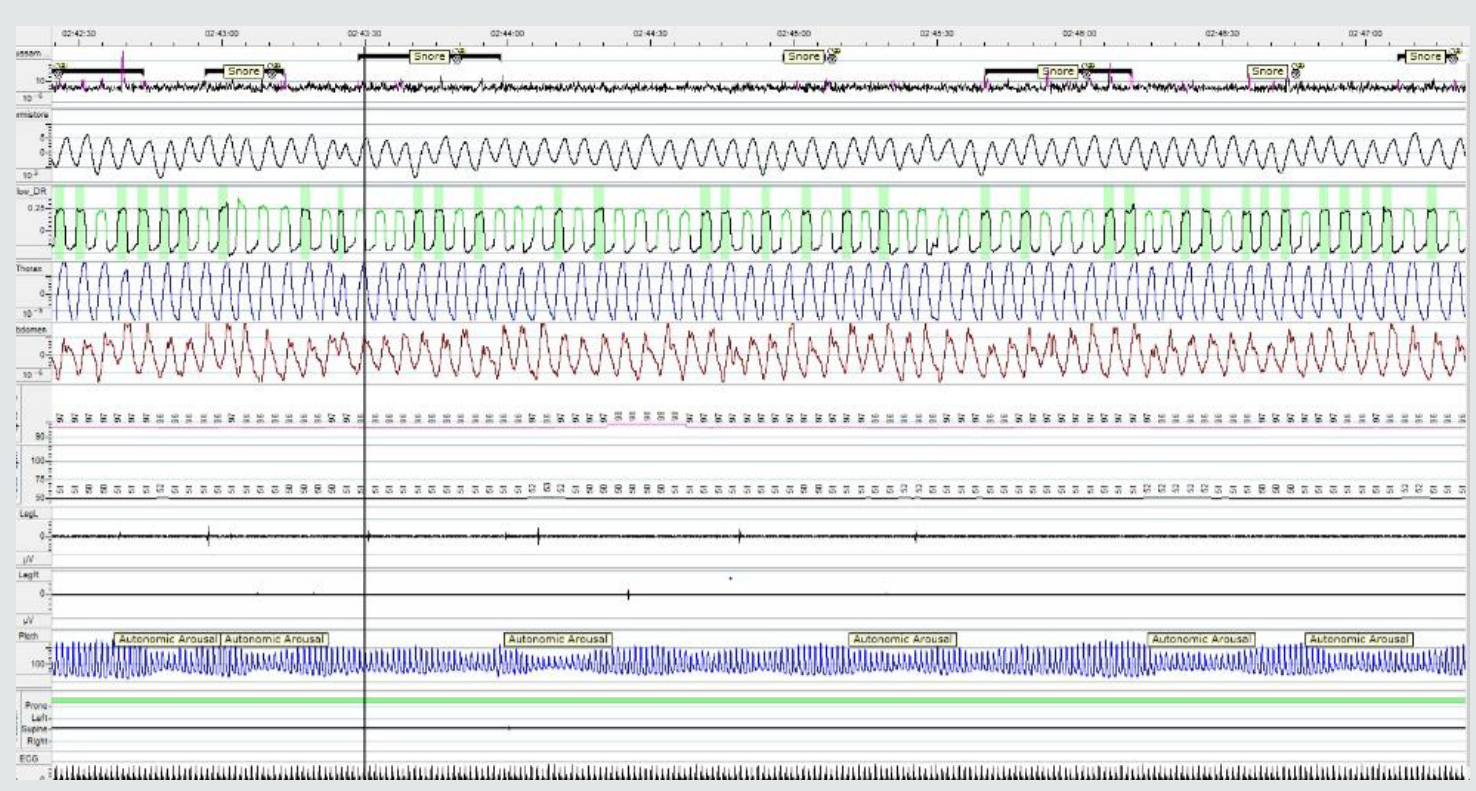

Figure 1: Basal polygraphic picture: flow limitations, absence of periodic movements; arousal index (AI) 49.7;heart rate variability (HRV) 4.1; Snoring 27.

HRV: LF/HF ratio,

LF (Low Frequency): Frequencies included between 0.04 and $0.15 \mathrm{~Hz}$.

HF (High Frequency): Frequencies included between 0.15 and $0.4 \mathrm{~Hz}$.

The LF band is considered mainly related to the activity of the sympathetic nervous system while the HF band is considered an expression of the parasympathetic activity.

The ratio between low and high frequencies (LF/HF) allows quantify the balance between the sympathetic and parasympathetic systems constituting the so-called

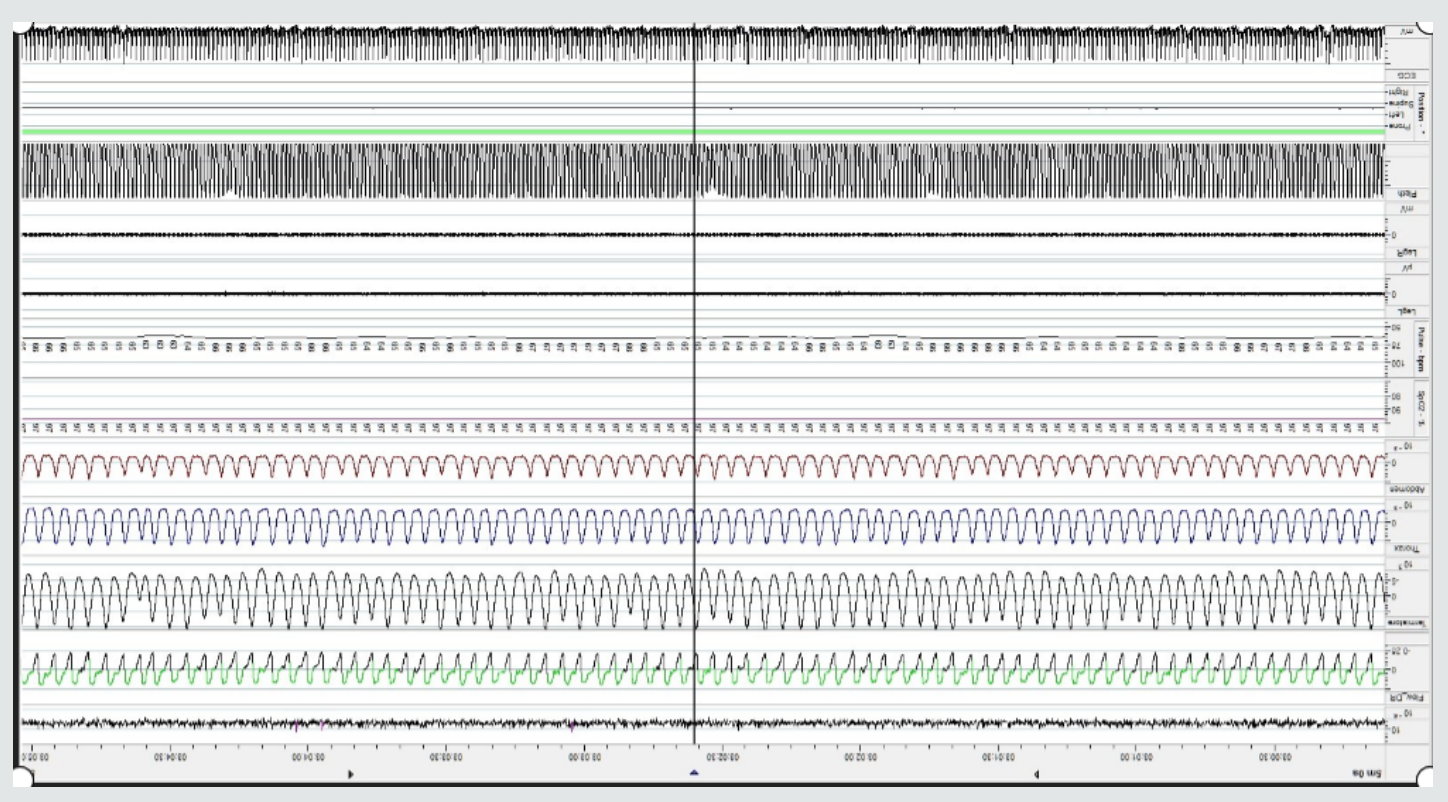

Figure 2: Polygraphic picture after one-month treatment with trazodone: resolution of the respiratory picture and normalization of the autonomic indices arousal index (AI) 11, heart rate variability (HRV) 1.3 Snoring $3 \%$. 


\section{References}

1. Castillo Vizuete JA, Sastre J, Del Cuvillo Bernal A (2019) Asthma, rhinitis, and nasal polyp multi morbidities. Arch Bronconeumol 55: 146-155.

2. Berry RB, Brooks R, Gamaldo C (2017) AASM Scoring Manual Updates for 2017 (Version 2.4). J Clin Sleep Med 13: 665-666.

3. Stöwhas AC, Lichtblau M, Bloch KE (2019) Obstructive Sleep Apnea Syndrome. Praxis (Bern 1994) 108: 111-117.
4. Heinzer RC, White DP, Jordan AS (2008) Trazodone increases arousal threshold in obstructive sleep apnea. Eur Respir J 31: 1308-1312.

5. Khouzam HR (2017) A review of trazodone use in psychiatric and medical conditions . Postgrad Med 129: 140-148.

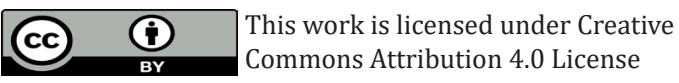

To Submit Your Article Click Here: Submit Article

DOI: $10.32474 /$ SJ0.2020.05.000214

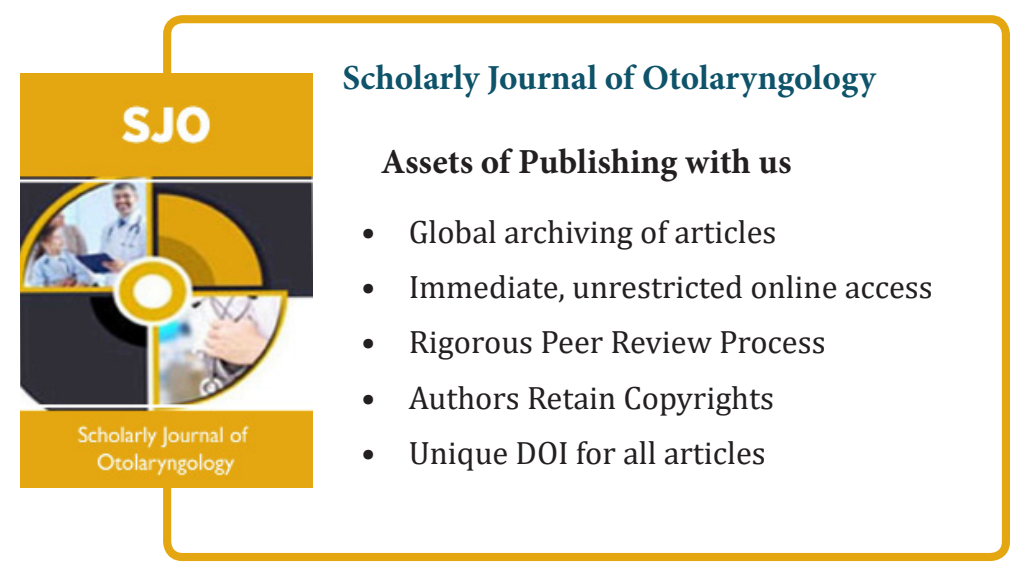

